\title{
Caracterización de los vínculos en la organización informal de una Institución de Educación Superior*
}

\author{
Characterisation of informal organisation links in a Higher Education Institution \\ Caracterização dos vínculos na organização informal de uma Instituição de Ensino Superior
}

Aura María Mena de la Cruz ${ }^{\text {a }}$

Universidad del Magdalena, Colombia

mamena@uninorte.edu.co

DOI: https://doi.org/10.11144/Javeriana.cao34.cvoiies

ORCID: https://orcid.org/0000-0003-3180-9470

Óscar García Vargas

Universidad del Magdalena, Colombia

ORCID: https://orcid.org/0000-0001-6332-0931

Recibido: 05 Abril 2021

Álvaro Zapata Dominguez

Universidad del Norte, Colombia

ORCID: https://orcid.org/0000-0001-8194-2259

\section{Resumen:}

Este artículo se propone caracterizar los vínculos en la organización informal identificada en tres dependencias de una Institución de Educación Superior de la región Caribe colombiana. El marco referencial comprende los conceptos sobre la organización informal y su estructura (integral, periférico, enlace, near-isolate, isolate). Se utilizó un enfoque metodológico de tipo descriptivo y se recolectaron 40 cuestionarios sociométrico válidos (datos mixtos). Los resultados muestran que los vínculos que sustentan estas relaciones informales en los grupos enfatizan en la convergencia de los valores personales que se comparten (por ejemplo, responsabilidad, honestidad, sinceridad); de igual manera, las caracterizaciones de los individuos enfatizan en cualidades (por ejemplo, amabilidad, colaboración, confiabilidad) como aspectos relevantes para la afiliación en relaciones informales en ellos.

Códigos JEL: J53, M14, M19.

Palabras clave: Organización informal, valores, vínculos, cualidades.

\section{Abstract:}

The purpose of this article is to characterise the informal organisation links identified in three dependencies of a Higher Education Institution in the Colombian Caribbean region. The referential framework includes the concepts of Informal Organisation and its structure (integral, peripheral, link, near-isolate, isolate). A descriptive methodological approach was used to collect a total of 40 valid socio-metric questionnaires (mixed data). The results show that the links that sustain these informal relationships in different groups lead to the convergence of shared personal values (for example, responsibility, honesty, sincerity). Similarly, the characterisations of individuals show that personal attributes (such as friendliness, willingness to collaborate, trustworthiness) are relevant when developing informal relationships within groups.

JEL Codes: J53, M14, M19.

Keywords: Informal organization, values, ties, qualities.

\section{Resumo:}

Este artigo tem como objetivo caracterizar os vínculos na organização informal identificada em três dependências de uma Instituição de Ensino Superior na região do Caribe colombiano. O quadro referencial inclui os conceitos sobre a organização informal e sua estrutura (integral, periférico, enlace, near-isolate, isolate). Uma abordagem metodológica descritiva foi utilizada e 40 questionários sociométricos válidos foram coletados (dados mistos). Os resultados mostram que os vínculos que sustentam essas relações informais nos grupos enfatizam a convergência de valores pessoais que são compartilhados (por exemplo, responsabilidade, honestidade, sinceridade); da mesma forma, as caracterizações dos indivíduos enfatizam as qualidades (por exemplo, gentileza, colaboração, confiabilidade) como aspectos relevantes para a afiliação em relacionamentos informais neles.

Códigos JEL: J53, M14, M19.

Notas de autor

$$
\text { a Autor de correspondencia. Correo electrónico: mamena@uninorte.edu.co }
$$


Palavras-chave: Organização informal, valores, vínculos, qualidades.

\section{Introducción}

Los seres humanos interactúan constantemente, generando relaciones sociales que inciden en el comportamiento individual y grupal. En el contexto organizacional se presentan dos tipologías de asociaciones humanas: la primera corresponde a aquellas agrupaciones de individuos que son creadas con el propósito de estructurar y cumplir roles que benefician a la dirección de la organización formal; mientras que la segunda, la asociación humana en el interior de la organización, surge como resultado de las interacciones sociales de los miembros de la organización en el lugar de trabajo. Dichas agrupaciones se caracterizan por la espontaneidad de las relaciones y porque estas no se encuentran prescritas por la organización formal; esta agrupación se denomina organización informal (Chanlat \& Bédard, 1990; De Faria, 2000; Higuita, Ballesteros \& Pérez, 2010; Mena \& Viloria, 2015; Chambi, 2017; Zehua, 2018; Marino, Dabos \& Gómez, 2020).

Las organizaciones se encuentran ramificadas por las relaciones que se dan tanto en sentido jerárquico, en concordancia con su organigrama, así como por las relaciones que se producen en la realidad social que conforman sus individuos. En la literatura sobre la organización informal se encuentra que este tipo de agrupación social cuenta con ciertos componentes estructurales que se cimientan en su unidad social básica (el grupo) y que, estos componentes se encuentran asociados a las relaciones de poder, el liderazgo, las formas de comunicación y la cooperación (Zapata, 2008; Higuita \& Leal, 2010; Miner, 2013; Mena \& Viloria, 2015; White, Currie \& Lockett, 2016; Chambi, 2017; Cross, 2018; Marino, Dabos \& Gómez, 2020). Sin embargo, la naturaleza de los vínculos que sustentan las relaciones en la organización informal se constituye en un aspecto pendiente por profundizar en el contexto organizacional.

Las instituciones de educación superior (IES) pueden ser consideradas como contextos organizacionales con una alta complejidad, dado que su estructura formalizada, la organización de sus funciones y los objetivos misionales que persiguen pueden generan relaciones sociales complejas y variadas (Bédard, 2004; Gaete \& Vásquez, 2008; Martínez, 2018). En este tipo de contexto organizacional se presentan también agrupaciones sociales de carácter informal que permean las relaciones entre sus miembros dentro de la estructura formal de la IES (Muñoz-Roja, Vargas-Ortiz, \& Latta-Arias, 2018; Chambi, 2017; Kegen, 2015; Winston, 2005; Heller \& Willower, 1968). En este sentido, este artículo tiene como objetivo realizar una descripción de los elementos característicos que sustentan las relaciones entre los miembros de la organización informal en algunas dependencias de una IES ubicada en la región caribe colombiana.

Bajo un enfoque metodológico de alcance descriptivo se realizó la recolección de los datos a partir de un cuestionario sociométrico que genera datos cualitativos y cuantitativos. Los resultados obtenidos reflejan que en las tres dependencias estudiadas ( $\mathrm{A}, \mathrm{B}$ y $\mathrm{C}$ ) efectivamente se presenta el fenómeno de la organización informal con un total de nueve unidades sociales identificadas dentro de estas -tres grupos por dependencia-. Sin embargo, cada dependencia tiene sus propias características de asociación entre las unidades sociales que se encuentran en esta. En términos generales, los miembros de la IES establecen un esquema de valores que caracteriza a la organización informal en su contexto y a sus relaciones, el cual se centra en cuatro valores fundamentales. De igual manera, los resultados reflejan que la asociación entre los individuos de la IES se encuentra relacionada con la percepción que se tiene de las cualidades de los miembros en la organización informal. 


\section{Fundamento teórico}

\section{La organización informal}

En la literatura sobre la gestión y las organizaciones se presentan diversas conceptualizaciones sobre el fenómeno de la organización informal; este concepto se deriva principalmente del enfoque de las Relaciones Humanas que define la organización informal como aquella que surge en contraposición a la prescripción que caracteriza a la organización formal. Así, la organización informal corresponde a una agrupación social producto de las interacciones de los miembros de la organización, cuya naturaleza es flexible y dinámica (Chanlat \& Bédard, 1990; Zapata, 2008; Higuita, Ballesteros \& Pérez, 2010; Chambi, 2017; Cross, 2018).

En el contexto organizacional, el fenómeno de la organización informal presenta un carácter relacional y sistémico, puesto que este tipo de agrupaciones sociales en el lugar de trabajo pueden conceptualizarse como un conjunto de contactos personales dentro de un sistema social dado, aunque en el seno de la organización informal estas relaciones personales se desligan de la estructura formalizada e idealizada de la organización (Johansen, 1992). En concordancia con lo anterior, una organización informal se entiende como una red derivada de los procesos de comunicación informal; esta red permite la transmisión de prácticas y otros contenidos además de poseer una cultura (Wang, Andrew \& Pavel, 2016).

Otra definición de organización informal expone que esta se constituye en una red social particular compuesta por una serie de dinámicas que generan una identificación y un deseo de pertenencia por parte de los miembros que componen la organización en general (De Faria, 2000). Las dinámicas sociales que se generan en la organización informal se derivan de las relaciones que establecen las personas, las cuales les permiten desarrollar elementos sociales como la comunicación, el liderazgo, la cooperación y el poder (Marino, Dabos \& Gómez, 2020; Zehua, 2018; White, Currie \& Lockett, 2016; Mena \& Viloria, 2015; Williams, Verwoord, Beery et al., 2013; Miner, 2013; Higuita \& Leal, 2010; Zapata, 2008; Laubach, 2005).

\section{Los vínculos en la organización informal}

La organización informal se encuentra compuesta por los diferentes tipos de relaciones que establecen los individuos a partir de las interacciones sociales que ellos generan en el interior de la organización. Las relaciones informales se definen a partir del vínculo que se pueden dar entre dos o más individuos, por lo que cada unidad social dentro de la estructura de la organización informal puede poseer diferentes tipos relaciones o grupos informales. Entre las tipologías de vínculos que sustentan las organizaciones informales se encuentran aquellos basados en el sentimiento de amistad, los que se basan en asesoría y consejos, los que se concentran en el aspecto político y la confianza, y las organizaciones informales cuyos vínculos se focalizan en los procesos de comunicación en el lugar de trabajo (Krackhardt \& Stern, 1988; Krackhardt, 1992; Krackhardt \& Hanson, 1993; Kuipers, 2009; Lopes, Guimarães, R., Lima et al., 2020; Marino, Dabos \& Gómez, 2020).

Otros lazos derivados de las relaciones sociales informales en la organización están asociados con la simpatía, la sociabilidad, la lealtad, el respeto y la confianza (Winston, 2005; Kuipers, 2009; Higuita \& Leal, 2010; Marqués, Farrerons, Arias et al., 2012; Venkataramani, Zhou, Wang et al., 2016). Estos vínculos son un elemento importante en las relaciones sociales que se encuentran en las agrupaciones sociales y permiten realizar interpretación de los comportamientos de los individuos a partir de las características asociadas a sus relaciones de intercambio. La fortaleza de dichos vínculos en las relaciones sociales se encuentra mediada por la combinación de elementos como el tiempo, la intensidad de las emociones, la confianza mutua e íntima y la reciprocidad (Granovetter, 1973; Santos, 1989; Lozares, 1996; Poveda, 2020). 
La relevancia de la naturaleza de los vínculos en la organización informal se encuentra en el contenido que se comparte e intercambia en las relaciones sociales que establecen sus miembros. En este sentido, la profundidad de los vínculos en una organización informal permite que sus miembros establezcan un sentimiento de unidad y cooperación, el cual se manifiesta usualmente a través del apoyo mutuo durante las labores en el lugar de trabajo. La organización informal tiene el potencial de beneficiar o entorpecer los procesos de la organización formal (Marino, Dabos \& Gómez, 2020; Lopes et al., 2020; Briggs, 2018; Mena \& Viloria, 2015; Sangrà \& Wheeler, 2013; Higuita, 2010; Zapata, 2008; Krackhardt \& Stern, 1988).

\section{Estructura social y organización informal}

La organización informal tiene la capacidad de desarrollar una estructura social que se manifiesta a lo largo de la organización formal. Dentro de esta estructura formal, algunos miembros se convierten en centrales o relevantes para sus unidades sociales, pero también para la estructura misma de la organización informal; esta posición central puede tener el poder para influenciar el rendimiento de los demás miembros de la organización. De igual manera, algunos miembros con posición central en la organización informal se convierten en líderes que inciden sobre el comportamiento de los demás, facilitando el consentimiento y el cumplimiento de las normas grupales informales (Chanlat \& Bédard, 1990; Laubach, 2005; Luria \& Berson, 2013; Johannessen, McArthur \& Jonassen, 2015; Zehua, 2018).

Una tipología de las posiciones en la estructura social de la organización informal señala cinco tipos de miembros: integral, periférico, enlace, near-isolate y el isolate (Heller \& Willower, 1968). De estos, dos son esenciales para la estructura social de la organización informal, el primero es el miembro integral debido a que este tiene la función de servir de soporte de las relaciones entre los diversos miembros de la unidad social (el grupo); el segundo corresponde al miembro enlace, quien es un individuo que pertenece a más de un grupo y tiene como función facilitar la conexión entre las diferentes unidades sociales que hacen parte de la estructura social de la organización informal.

Por su parte, los miembros periféricos, near-isolate y el isolate se caracterizan por una débil asociación con los miembros que componen la estructura grupal. El miembro periférico es aquel que posee una asociación débil con alguno de los miembros que hacen parte de la estructura social del grupo. El miembro de tipo nearisolate es un individuo que se encuentra débilmente asociado con otros miembros del grupo por relaciones no recíprocas, es decir, emite referencias, pero no recibe de otros. Por último, el miembro isolate hace referencia a aquellos individuos aislados dentro de la estructura social o que carecen de relación con otros miembros (Heller \& Willower, 1968; Zehua, 2018).

Otra tipología sobre la estructura social de la organización informal corresponde a la clasificación de las unidades sociales que la componen. Esta tipología establece dos categorías posicionales relevantes: la primera es el clan administrativo que se caracteriza por una fuerte asociación, ventajas e influencias sobre la organización formal; y la segunda es la periferia informal que se caracteriza por una baja capacidad para influir sobre la organización formal, pero que posee fuerte relaciones sociales entre sus miembros (Laubach, 2005).

\section{Metodología}

Este artículo presenta un enfoque metodológico de carácter descriptivo; la fuente de datos de la investigación que lo origina es de tipo primaria y fue recolectada a partir de un cuestionario sociométrico compuesto por una matriz de referenciación $3 \times 3$. El cuestionario se aplicó a los miembros de tres dependencias (tanto funcionarios como docentes) de la organización objeto de estudio, la cual fue una institución de educación superior ubicada en el caribe colombiano; en el proceso se recolectó un total de 40 cuestionarios válidos. 
El instrumento se aplicó en las áreas permitidas por la IES para la realización del estudio, para efectos del procesamiento de los datos, estas fueron clasificada según actividad funcional (docencia, investigación y gestión administrativa) de la IES dejando como resultado un total de tres dependencias rotuladas como A, B y C. La dependencia A corresponde a una de las áreas formación que posee la IES, está compuesta por personal docente de niveles de pregrado (modalidad presencial, virtual, distancia), posgrado y cursos complementarios, y el personal administrativo que se divide entre asistencia administrativa y docentes con roles administrativo. Por su parte, la dependencia B hace parte de la estructura de investigación, esta área se encuentra compuesta por personal de investigación, docentes con rol de investigadores y el personal administrativo de apoyo a la investigación. La dependencia $\mathrm{C}$ comprende una de las áreas funcionales administrativas de la IES, está integrada por personal administrativo orientado a procesos contables y financieros.

El instrumento utilizado comprende una adaptación del cuestionario sociométrico de Winston (2005); la composición del cuestionario corresponde a una matriz donde cada encuestado debe referenciar a tres individuos (nombre, cargo, dependencia) de la organización objeto de estudio que considera como sujetos con quien sostiene relaciones informales en el entorno organizacional. El cuestionario plantea adicionalmente una pregunta abierta que se orienta a caracterizar a las personas $(\mathrm{A}, \mathrm{B}, \mathrm{y} \mathrm{C})$ referenciadas por el encuestado, particularmente frente a sus cualidades (personalidad, valores, competencias).

El cuestionario sociométrico es una herramienta de recolección de información que tiene como finalidad la evaluación de la preferencia social, el atractivo interpersonal y la dirección de las relaciones que se desarrollan entre los individuos que pertenecen a una agrupación social; esta técnica se considera una medida muy válida y se encuentra basada principalmente en la información autor-informada (Winston, 2005).

El resultado de la matriz de referenciación del cuestionario aplicado es una representación gráfica denominada sociograma, la cual muestra las conexiones que se dan entre los individuos que pertenecen a la agrupación social de carácter informal. Los datos cuantitativos recolectados con el instrumento sociométrico se procesaron en el software AGNA; el cual, además de generar el sociograma, presenta una serie de variables tales como la cohesión y la densidad (ver tabla 1 ).

TABLA 1

Definición de variables descriptivas del sociograma

\begin{tabular}{ll}
\hline \multicolumn{1}{c}{ Variables } & \multicolumn{1}{c}{ Definición } \\
\hline Nodo & Cada uno de los individuos dentro del sociograma \\
Edge & Lazos o relaciones entre nodos (individuos) \\
Densidad & $\begin{array}{l}\text { El grado en que todas las posibles interacciones están } \\
\text { presentes }\end{array}$ \\
Cohesión & La fuerza de los lazos o las relaciones \\
Recepción & Veces que es referenciado por otro individuo (nodo) \\
Emisión & Veces que un individuo referencia a otros \\
\hline & Fuente: elaboración propia.
\end{tabular}

Por otra parte, los datos de carácter cualitativo (opiniones y percepciones) derivados de la pregunta abierta del cuestionario fueron analizados a partir de la comparación entre las respuestas de los individuos y las categorías convergentes que se consignaron en tablas descriptivas. Para realizar el estudio se tuvieron limitaciones en cuanto al acceso a información sensible, indisposición de varios participantes para ser encuestados, formularios incompletos, limitación en el tiempo y espacio para aplicar el instrumento en la institución, limitaciones de recursos financieros para continuar con otras fases del proyecto. Es por ello que el alcance del estudio enfatiza en lo descriptivo. 


\section{Resultados}

La organización informal en la institución objeto de estudio presenta un conjunto de nueve unidades sociales (grupos) distribuidas entre las tres dependencias estudiadas. La tabla 2 muestra que la dependencia A está compuesta por un total de 25 nodos y 48 Edges (relaciones) y es en ella en la que se presenta el mayor número de referenciaciones para un individuo; la dependencia $B$ presenta un total de 22 nodos y 40 Edges; por último, la dependencia $C$ presenta 14 nodos y 18 Edges, por lo que, en ella es donde se presentan las unidades sociales más pequeñas de la estructura de la organización informal, aunque sus valores de cohesión y densidad son más compactos, lo que suele ocurrir en la composición de grupos pequeños.

En un análisis general de los individuos que componen las unidades sociales de la organización informal se encontraron 102 cualidades, entre caracterizaciones de personalidad y valores. El esquema de valores que presenta la organización informal en el contexto de la IES se define a partir de valores como responsabilidad, honestidad, respeto y sinceridad; en menor medida se presentan la solidaridad y la lealtad. Los valores como la responsabilidad, el respeto, la honestidad y la lealtad se encuentran en concordancia con los valores institucionales de la IES.

TABLA 2

Datos sociométricos descriptivos generales por dependencia

\begin{tabular}{l|c|c|c}
\hline \multicolumn{1}{c|}{ Variable } & Dependencia A & Dependencia B & Dependencia C \\
\hline Nodos & 25 & 22 & 14 \\
\hline Edges & 48 & 40 & 18 \\
\hline Recepción & Mín. 1 - Máx. 6 & Mín. 1 - Máx. 5 & Mín.1 - Máx. 3 \\
\hline Emisión & Mín.1 - Máx. 3 & Mín. 2- Máx. 3 & Mín. 3 - Máx. 3 \\
\hline Cohesión & 0,02666 & 0,038961038 & 0,043956045 \\
\hline Densidad & 0,08 & 0,08658009 & 0,0989011 \\
\hline
\end{tabular}

Fuente: elaboración propia.

Adicionalmente, los encuestados realizaron caracterizaciones muy específicas de la personalidad y las cualidades de los miembros de la organización informal con la que se identifican. Entre las características más representativas se encuentran el ser amigable, la amabilidad, la colaboración, el compañerismo, la seriedad, la confiabilidad, la alegría, el buen sentido del humor, la inteligencia, el profesionalismo, la prudencia, ser cariñoso, tener compromiso, ser sociable y ser trabajador. Otras características expresadas, aunque en menor medida, son la proactividad, la transparencia, la empatía, ser servicial y la humildad.

\section{Hallazgos dependencia A}

En esta dependencia se identificaron tres unidades sociales que hacen parte de la organización informal de la IES en conjunto. La figura 1 muestra dos miembros de la organización informal que se ubican en la posición de enlace, estos son el sujeto S2 y S10, estos dos sujetos permiten la conexión entre las tres unidades sociales de la dependencia A. Vale destacar que el S10 se ubica dentro de la estructura formal como el director de la dependencia, pero el S2 tiene mayor número de referenciaciones (aunque tiene un cargo de coordinador).

Estos miembros de enlace se encuentran en concordancia con el esquema de valores que presenta la organización informal, dado que, tanto el $\mathrm{S} 10$ como el S2 son caracterizados con los valores de responsabilidad y respeto; de igual manera, se le asocian cualidades como amigable, amabilidad, inteligencia y profesionalismo. Aunque los sujetos S10 y S2 se encuentran en posiciones de autoridad en la estructura organizacional de la IES, en la figura 1 se destacan otros dos sujetos -S24 y S1 coordinadores- como miembros relevantes para la composición de la organización informal. 


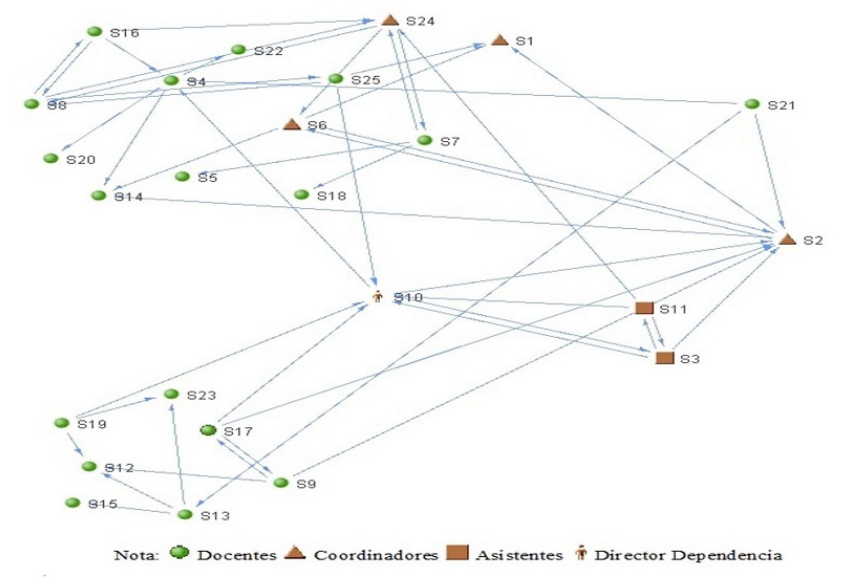

FIGURA 1

Socio-grama de la dependencia A

Fuente: elaboración propia.

Otros individuos referenciados que se ubicaron como miembros integrales en las unidades sociales de la organización informal, ubicada en esta dependencia, son los sujetos S8, S4, S14 y S12, los cuales dentro de la estructura organizacional se ubican en cargos de docentes.

En la figura 1 se observa que la primera unidad social identificada se encuentra compuesta por un total de 13 nodos (S1, S4, S5, S6, S7, S8, S14, S16, S18, S20, S22, S24, S25); entre los cuales varios miembros presentan referenciación bilateral, como es el caso de los sujetos S7 y S24, y la relación del miembro integral S8 con los sujetos S24, S25 y S16. Esta unidad social se caracteriza porque concentra el mayor número de los miembros integrales que componen la organización informal en esta dependencia con un total de 5.

Esta unidad social se encuentra en conexión con las otras unidades sociales a través de los dos miembros enlace de la organización; sin embargo, la figura 1 muestra también que esta unidad social tiene una relación bilateral entre el miembro S6 con el miembro enlace S2. En cuanto a la percepción que tienen los demás miembros de la organización informal sobre los miembros de tipo integral en esta primera unidad, se evidencia una concentración de las relaciones en torno a las características de la personalidad como el conocimiento, la prudencia, la amistad y el compañerismo (ver tabla A1).

La segunda unidad social identificada en la figura 1 se caracteriza por ser una diada conformada por personal administrativo de tipo asistencial que no establecen relaciones con personal docente, sino solo con aquellos docentes con carga administrativa (director y coordinadores). Esta unidad está compuesta por los sujetos S11 y S3, quienes tienen una relación bilateral de referenciación, y el miembro S3 presenta una relación bilateral con el miembro enlace S10. En cuanto a la caracterización, el sujeto S3 define al sujeto S11 como "es amigable, inteligente y atenta", mientras que el sujeto $S 11$ caracteriza al sujeto S3 como "es mi compañera, es responsable, sincera y humilde". Las caracterizaciones anteriores permiten considerar que esta díada presenta una fuerte asociación que transciende de la relación formal de compartir el puesto de trabajo, dado que ambas se consideran dentro de la organización informal de la otra.

Por último, la figura 1 muestra que la tercera unidad social de la organización informal identificada en esta dependencia se encuentra compuesta por un total de siete nodos (S23, S12, S19, S15, S13, S9, S17), de los cuales solo uno es miembro integral (sujeto S12). De igual manera, se observa una referenciación de tipo bilateral entre los miembros S9 y S17. Estos dos miembros consideran que su relación se encuentra basada en la amistad pues en la descripción que el miembro S9 hace sobre S17 señala que ambos son amigos desde que estudiaron el pregrado, S9 destaca la personalidad noble de S17.

Por otro lado, el sujeto $S 12$, que se constituye en el miembro integral, no presenta emisión de referencias hacia otros miembros de esta unidad social. Sin embargo, la caracterización de este miembro se encuentra en concordancia con el esquema de valores que comparte la organización informal, dado que presenta las descripciones realizadas por los sujetos S19 y S13, que le identifican los valores de respeto, sinceridad y responsabilidad. De igual manera, estos sujetos S19 y S13 en su descripción de S12 manifiestan que cuenta con 
características de personalidad como ser amigable, sociable y la amabilidad que predominan en la organización informal.

La conexión con los otros grupos se da a través de los miembros enlaces $S 10$ y $S 2$, los cuales son referenciados por los miembros S9, S17 y S19 de esta tercera unidad social de la organización informal en esta dependencia. Esta unidad a diferencia de las otras dos no presenta referenciación bilateral con los miembros enlaces (ver figura 1). Se observa también que ciertos nodos de la organización informal en la dependencia A presentan una o dos referenciaciones que los clasifica en la categoría de periférico de Heller y Willower (1968); solo un miembro clasifica en la categoría de near-isolate, el sujeto S21 (ver figura 1). Los resultados indican que los docentes y el personal administrativo de tipo asistencial no se relacionan de manera informal, lo que puede responder una percepción por parte estos individuos de mantener la distancia formal que representa la rigidez de la estructura organizacional en este contexto de la IES, y en el caso específico de los docentes, estos son más abiertos a relaciones informales con los docentes con roles administrativo dado que comparten las dinámicas de enseñanza y formación en los programas y estos cargos tienden a ser rotativos.

\section{Hallazgos dependencia B}

En cuanto a los resultados obtenidos para la dependencia B, la figura 2 presenta un total de tres unidades sociales que están poco conectadas entre ellas; no se evidencia un miembro de enlace que permita la conexión entre las unidades sociales de la organización informal en esta dependencia. Esta situación se podría explicar por las diferentes líneas de investigación o disciplinas que se encuentra en esta dependencia, puesto que no todos los docentes investigadores pertenecen a un mismo programa o facultad. A diferencia de las unidades sociales de la organización informal en la dependencia A que sí cuentan con miembros de enlaces, aunque todas las unidades se limiten al espacio organizacional de la dependencia A (ver figura 1). Las unidades sociales de la dependencia B se encuentran conectadas con otros sujetos que pertenecen a otras áreas o dependencias de la IES.

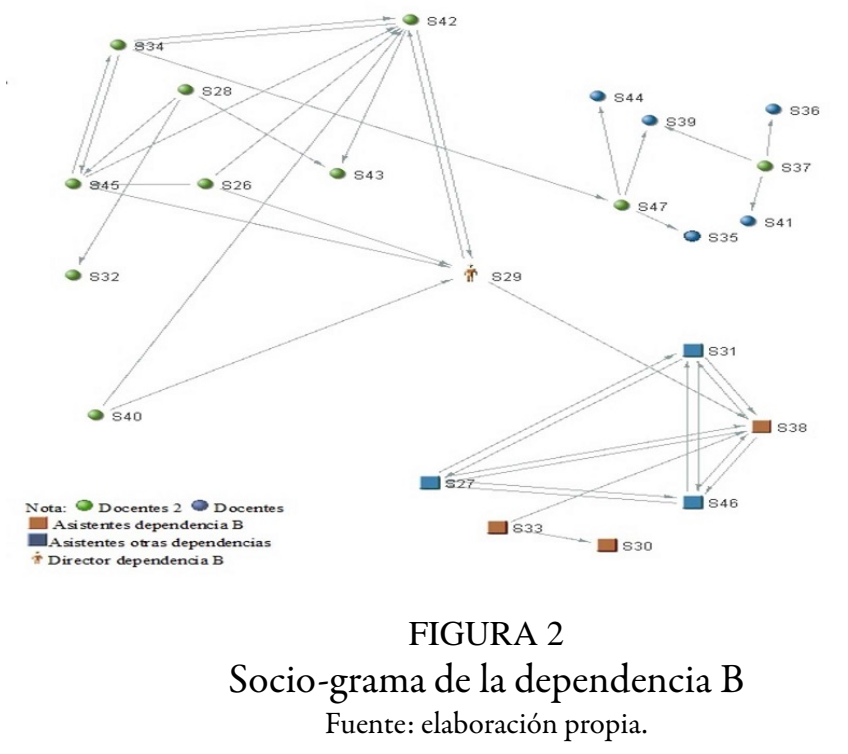

La dependencia B está compuesta por individuos que ostentan diversos roles o cargos en la estructura jerárquica formal; en concreto, hay una división del rol de personal docente en dos tipos, en los cuales el tipo 2 presenta unas funciones adicionales al docente 1 y que están orientadas específicamente al cumplimiento de uno de los objetivos misionales de la IES. La figura 2 presenta una diferenciación clara de las unidades sociales que componen la organización informal en esta dependencia, puesto que los individuos se han asociado a partir de sus roles en la estructura formal; por esto se observa una unidad social compuesta por docentes tipo 2 , una unidad social de personal administrativo, y una unidad social de docentes tipo 1 y tipo 2. 
La primera unidad social de la organización informal en la dependencia B está compuesta por nueve nodos (S34, S28, S45, S26, S43, S32, S42, S29, S40) que corresponden a miembros que ocupan el rol de docentes tipo 2 , y en ella se encuentra el director de la dependencia; un aspecto relevante de esta unidad social es que tanto el miembro S29 (director dependencia) como el S42 (docente tipo 2) resultan ser miembros integrales, pero el sujeto S42 presenta un mayor número de referenciaciones que el sujeto S29. Esta unidad emite referencias hacia las otras dos unidades sociales de la organización informal en esta dependencia, pero estas conexiones no alcanzan el nivel de enlace, dado que no hay referencia por parte de las otras unidades hacia miembros de esta unidad (ver figura 2).

Esta primera unidad social muestra un total de tres referenciaciones bilaterales que se producen entre el sujeto $S 45$ con el sujeto S34, el sujeto S34 con el sujeto S42, y el sujeto S42 con el sujeto S29. De estas combinaciones de relaciones bilaterales se destacan como miembros integrales los sujetos S42, S29 y S45 (ver figura 2). Las caracterizaciones de los miembros integrales para esta primera unidad social de la organización informal en la dependencia $B$ refleja que estos individuos centrales presentan una similitud en los valores que perciben los otros miembros en ellos, como son los de responsabilidad, respeto y sinceridad. En cuanto a las características de personalidad, estos miembros integrales se destacan en los aspectos como la inteligencia y el don de gente (ver tabla A2).

Por otro lado, la segunda unidad social de la organización informal en la dependencia B se encuentra compuesta por seis nodos que corresponden a los sujetos S38, S27, S31, S46, S30 y S33; un aspecto relevante en la conformación de esta unidad se encuentra en que los cuatro miembros integrales (S38, S27, S31 y S46) presentan referenciación bilateral entre ellos (ver figura 2). El sujeto S38 corresponde a un miembro con rol administrativo asistencial que presentan un número mayor de referenciaciones que el sujeto S29 (quien es su jefe inmediato), el cual también referencia a este sujeto; aunque este sujeto S38 es referenciado por sus compañeras en el lugar de trabajo, sus relaciones informales transcienden la dependencia en la que se encuentra adscrita a otras.

El sujeto S38 es caracterizado por sus compañeros de dependencia a partir de sus cualidades expresadas en el lugar de trabajo que son el carisma y su productividad (ver tabla 2). Por otro lado, la similitud de las características y cualidades de los miembros integrales en la segunda unidad social de la organización informal en la dependencia B se concentran en un esquema de valores compuestos por el respeto y la sinceridad; mientras que las características de personalidad más relevantes en esta unidad social son el ser cariñoso, ser trabajador, la amabilidad, la humildad, el carisma y la colaboración (ver tabla A2). En esta unidad social ningún miembro referencia a miembros de las otras unidades de la dependencia $\mathrm{B}$, el vínculo con la primera unidad social de la dependencia B se encuentra por la relación de emisión que desde el sujeto S29.

Por último, la tercera unidad social que se manifiesta en la figura 2 se encuentra conformada por un total de siete nodos (S47, S44, S39, S36, S41, S35, S37), de los cuales dos miembros se encuentran adscritos a la dependencia $B$ mientras que los otros cinco referenciados se vinculan a otra dependencia. Esta unidad presenta una conexión con la primera unidad social de la organización informal de la dependencia B por la emisión del sujeto $S 34$ que referencia al sujeto $\$ 47$, sin embargo, ningún miembro de esta tercera unidad referencia algún miembro de la primera unidad. Los miembros que componen este grupo presentan una afiliación asociada con la profesión que comparten.

En esta unidad, el sujeto S39 pertenece a otra dependencia, pero este miembro recibe al menos dos referencias que provienen de los sujetos S47 y S37, quienes están adscritos a la dependencia B como docente tipo 2; el sujeto S39 es caracterizado por el sujeto $S 47$ con las siguientes cualidades "transparencia, lealtad, ética, profesionalismo"; mientras que para el sujeto S37 la relación con el sujeto S39 gira en torno a la confianza por la relación de amistad entre ambos. Este sujeto S39 se puede considerar como un miembro importante para la estructura de esta unidad, pero no alcanza el nivel de miembro integral. Por otra parte, al igual que en la dependencia A, la B presenta nodos con una o dos referenciaciones que los ubica en las categorías de 
periférico, e inclusive dos de ellos (S40 y S33) pueden considerarse dentro de la categoría near-isolate (Heller \& Willower, 1968).

En concordancia con los resultados de la dependencia A, los resultados muestran que los docentes investigadores y el personal administrativo de tipo asistencial no se relacionan de manera informal y mantienen su distancia jerárquica en la estructura organizacional, esto puede estar relacionado con el hecho de que en esta dependencia en particular, los docentes de investigación no permanecen de forma constante en el espacio de trabajo dado que se encuentran asociados a otras áreas como las facultades o se encuentran realizando actividades de investigación fuera del entorno de la IES, además que el espacio de la dependencia $\mathrm{B}$ es dispuesto por los docentes investigadores para desarrollar sus actividades de escritura, procesamiento de datos o para asistencia a las reuniones con el director.

\section{Hallazgos dependencia C}

En cuanto a los resultados obtenidos para esta dependencia, la figura 3 muestra tres unidades sociales que interactúan poco una de con la otra; inclusive una de estas unidades se constituye un grupo aislado de los demás, compuesto principalmente por miembros con rol o posición de autoridad en la estructura formal de la IES. La primera unidad social de la organización informal que se identifica en la dependencia $\mathrm{C}$ corresponde a un conjunto de contactos de cuatro nodos (S50, S56, S49, S59); donde un solo individuo referencia atros tres.

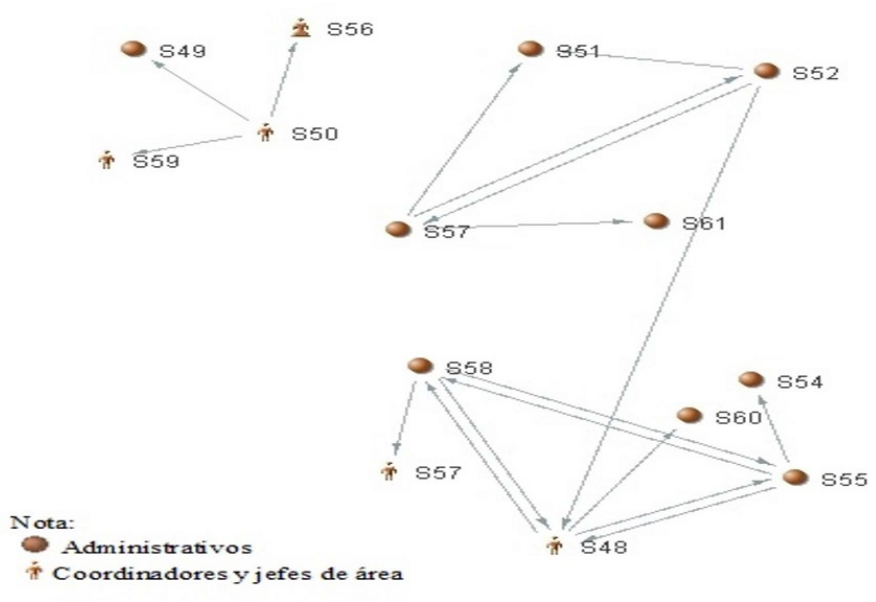

FIGURA 3

Socio-grama de la dependencia C

Fuente: elaboración propia.

En cuanto a las caracterizaciones de los individuos que componen esta unidad social, solo se aprecian dos descripciones que caracterizan a los sujetos S59 y S49; la descripción que realiza el S50 sobre el sujeto S59 expresa que este individuo se caracteriza por "su profesionalismo y dinamismo"; mientras que la caracterización que hace el sujeto S50 del sujeto S49 señala que el sujeto S49 se destaca por su "seriedad, responsabilidad". Como se observa en la figura 3, esta unidad se encuentra conformada por miembros con cargo de coordinador o jefe, por lo que esta unidad se puede categorizar en la tipología de organización informal como clan administrativo (Laubach, 2005).

La segunda unidad social de la organización informal en la dependencia $\mathrm{C}$ se encuentra compuesta por cuatro nodos (S51, S52, S57, S61) que presentan una referencia bilateral y una referencia en común entre los miembros emisores (ver figura 3). De igual manera uno de los nodos emisores (sujeto S52) se conecta de forma unilateral con la tercera unidad social identificada en la organización informal de la dependencia C. Por otro lado, el sujeto $S 52$ presenta referenciación bilateral con el sujeto S57; entre los valores asociados a esta relación bilateral entre sujetos se encuentra que el sujeto S57 considera que el sujeto S52 "es una persona sincera, 
correcta, afinidad de temáticas", mientras que el sujeto S52 considera al sujeto S57 como "emprendedora, alegre, curiosa y creativa".

Las caracterizaciones anteriores de los sujetos S57 y S2 se distancian en cierta medida de los puntos de convergencias de los individuos que pertenecen a la organización informal de la IES. Sin embargo, al nodo al que corresponde el sujeto $S 51$, el cual posee el mayor número de referencias en este grupo (ver figura 3), se le atribuyen valores y cualidades como "madura, seria, responsable (S57) ...paciente, responsable, servicial, tolerante (S52)”. Las descripciones realizadas sobre el sujeto S51 muestran que el valor más característico corresponde a la responsabilidad, el cual se encuentra en concordancia con el esquema general de valores de la organización informal en la IES.

Por último, la tercera unidad social identificada de la organización informal en la dependencia C corresponde al conjunto de siete nodos (S58, S53, S48, S55, S60, S54). Esta unidad presenta el único individuo con el número de referenciación de tres para ser considerado integral, de igual manera este mismo sujeto $S 48$ es referenciado por el sujeto $S 52$ que pertenece a la segunda unidad social. Los miembros de este grupo informal se caracterizan por su fuerte referenciación bilateral, por lo que se evidencia una fuerte relación en tres miembros específicos, sujeto $S 48$ (coordinador de área), sujeto S58 y sujeto S55. Como estos tres sujetos se referencian entre ellos, en sus emisiones solo quedan unos miembros que no tienen relaciones con otros siendo por ello miembros periféricos.

De acuerdo con las descripciones de los sujetos S58 y S52, el miembro integral S48 se caracteriza por los valores de responsabilidad y la honestidad; y en cuanto a sus cualidades, ellos mismos destacan la confianza, la colaboración, su capacidad de organización y que es un sujeto pasivo. Por su parte, el sujeto $S 55$ tiene una relación bilateral con el sujeto $S 48$ y con el sujeto $S 58$, la caracterización que realiza el sujeto $S 58$ sobre el sujeto S55 es que es una persona "responsable y honesta"; mientras que el miembro integral sujeto S48 expone que el sujeto $S 55$ es una persona "sociable, alegre y chistosa". De igual manera, el sujeto $S 58$ presenta una relación bilateral con los sujetos S55 y S48, pero solo el sujeto S48 menciona las cualidades del S58 en términos de "sinceridad, empatía y responsabilidad".

\section{Discusión}

Los resultados obtenidos en este estudio sobre las relaciones entre los miembros de la organización informal en una IES del Caribe colombiano permiten observar que en este contexto organizacional se presentan las siguientes cuatro categorías de la tipología de miembros de la organización informal: miembros integrales, enlace, periféricos y near-isolate (Heller \& Willower, 1968). A pesar de que los resultados reflejan individuos con posiciones centrales -miembros integrales y enlaces-, estos sujetos no fueron caracterizados de forma directa o precisa como líderes para la organización informal en las dependencias estudiadas.

En la dependencia A un solo miembro de tipo enlace fue descrito por otro sujeto como líder, por lo que en este contexto específico de la IES la configuración de la estructura social de la organización informal considera otros elementos para otorgar centralidad a los individuos, como es el caso de cualidades como la humildad y la inteligencia, junto con la cercanía con el esquema de valores que maneja la agrupación en general y permite la identificación social. En concordancia con lo anterior, los líderes de la organización informal son definidos como individuos que se ajustan de mejor manera a los valores y creencias que dominan la agrupación social, estos están caracterizados por un fuerte sentido de la palabra y por el ejercicio de cualidades como el altruismo y la humildad (Chanlat \& Bédard, 1990; Miner, 2013; Williams et al., 2013; White, Currie \& Lockett, 2016).

En cuanto a la tipología de la posición de las unidades sociales en la estructura de la organización informal, compuesta por las categorías clan administrativo y periferia informal (Laubach, 2005), los resultados no permiten ubicar con precisión las unidades sociales identificadas en las dependencias en estas tipologías dado que no se evidencia en las descripciones ni en los datos el nivel de efectos (ventajas y beneficios) de estas 
unidades sociales informales sobre sus miembros en la organización formal. Sin embargo, podría considerarse que la identificación de los vínculos en las organizaciones informales puede contribuir a que los directivos de las organizaciones tengan una precisión cognitiva sobre las características de los individuos que componen estas unidades sociales, de manera que, frente a la implementación de iniciativas se establezcan elementos de juicio frente efectos resistencia-contraposición o beneficios de estas redes sociales al interior de la organización (Marino, Dabos \& Gómez, 2020; Brigg, 2018; Cross, 2018).

La trayectoria de los vínculos identificados en las dependencias de la IES estudiada muestra dos tipos de conexiones: (i) unidireccionales, en las unidades sociales graficadas en el socio-gramas se presentan nodos que emiten o reciben en una sola dirección; y (ii) bidireccional evidenciadas en las unidades sociales más compactas y pequeñas. Los lazos unidireccionales no implican un intercambio unilateral en un contexto real de las organizaciones, pueden más bien conexiones débiles pero beneficiosas en el sentido de posibilitar flujo de información y servir de conectores con otros grupos u otras partes de la organización (Granovetter, 1973; Briggs, 2018; Cross, 2018; Poveda, 2020).

De igual manera, las descripciones y caracterizaciones acerca de los miembros de la organización informal ubicados en las dependencias de la IES muestran patrones de afiliación que se encuentran en concordancia con las tipologías de organizaciones informales presentes en la literatura como organizacionales informales basadas en amistad, la política, la confianza y la comunicación (Krackhardt \& Stern, 1988; Krackhardt, 1992; Krackhardt \& Hanson, 1993; Kuipers, 2009; Zehua, 2018; Lopes et al., 2020). En el contexto organizacional específico de la IES, la conformación de organizaciones informales se encuentran en torno a la amistad, la confianza y las cualidades asociadas al lugar de trabajo, siendo la amistad el elemento más característico de las relaciones informales en el lugar de trabajo.

La relevancia de la caracterización del ser amigable en la organización informal identificada en el contexto específico de la IES, y la descripción de los valores en las unidades sociales, permite considerar la relevancia de los vínculos de la amistad para la conformación de unidades sociales de tipo informal, debido a que, estos vínculos se caracteriza por proveer confianza o apoyo emocional generando una fuerte afiliación que les permiten llegar a acuerdos culturales e, inclusive, a configurar una cultura propia (Krackhardt \& Stern, 1988; Krackhardt \& Kilduff, 2002; Winston, 2005; Zehua, 2018; Poveda, 2020).

Estos vínculos de amistad en unidades organizativas como la dependencia B (área de investigación de la IES), pueden contribuir con la generación de espacios para la transferencia de conocimientos e innovación (Capone \& Lazzeretti, 2018; Zehua, 2018). Por otro lado, el énfasis de los valores en las caracterizaciones y descripciones de los miembros que conforman la organización informal permiten considerar el carácter cultural de los vínculos establecidos por estos individuos en el contexto organizacional específico de esta IES, puesto que los vínculos en la organización informal pueden transmitir conocimiento asociado con la cultura, como los valores culturales que comparten una determinada agrupación social (Krackhardt \& Hanson, 1993; Winston, 2005; Wang, Andrew \& Pavel, 2016).

\section{Conclusiones}

Las descripciones realizadas en el estudio corresponden a la naturaleza del concepto de organización informal, fenómeno que no es ajeno a las IES puesto que sus miembros pueden generar afiliaciones o vínculos de tipo informal en las dependencias a las que se encuentras adscrito. En el contexto de la IES estudiada, las relaciones entre el personal docente y el personal que realiza labores administrativas de forma asistencial son distantes, aspecto que se aborda de forma limitada en la investigación, pero que se sugiere para desarrollar en futuras investigaciones.

Los resultados obtenidos pueden ser considerados como una exploración inicial de los valores que sustentan la dinámica social y la cultura en la IES de estudio, que podría ser ampliado en futuras investigaciones sobre 
la organización informal y la cultura organizacional en IES. Por otro lado, el vínculo basado en la amistad se encuentra presentan en las tres dependencias estudiadas, lo que respalda la literatura revisada sobre la organización informal. La amistad y otros vínculos en el contexto organizacional de las IES pueden ser considerados en futuros estudios sobre las relaciones informales y la gestión del conocimiento en este tipo de instituciones, con miras a ampliar la naturaleza de la organización informal en dependencias como la B (investigación).

Por último, los resultados obtenidos son limitados frente a la amplitud de la organización informal entendida como una red social. El estudio no permite identificar con precisión los efectos que pueden generar estas unidades sociales en la IES, por lo que no se pueden establecer posiciones dentro de la estructura de la organización como clan administrativo o periferia informal, tampoco se precisa sobre el nivel de conciencia de los directivos sobre el tejido de relaciones informales en estas tres dependencias de la IES, dado que, el estudio solo se limita a la conciencia de los directivos sobre sus propias relaciones informales, además que no se realizan descripciones sobre efectos de resistencia o beneficio de estas unidades sociales en el entorno de la IES. Lo anterior puede considerarse como aspectos interesantes para ser abordados en futuras investigaciones en las particularidades de este contexto específico, incluso a nivel comparativo con IES de otros territorios o países.

\section{Consideraciones éticas}

La investigación de la cual deriva el presente artículo no requirió aval del comité ético de la institución universitaria financiadora.

\section{Contribución de los autores}

La mayor contribución del autor de correspondencia consistió en la adaptación de los resultados de la investigación a la estructura de artículo. El segundo autor contribuyó en la amplitud de los análisis y comprensión de las dinámicas del entorno del caso. De igual manera, el tercer autor aportó a la construcción del artículo su capacidad analítica y experiencia en el marco referencial de la organización informal y la dinámica del entorno del caso.

\section{Financiación}

Artículo de investigación derivado de la investigación titulada El grupo informal y la cultura organizacional: una perspectiva del hombre y la realidad social dentro de las organizaciones, financiado por la Universidad del Magdalena, Santa Marta, Colombia.

\section{Conflictos de interés}

Los autores no tienen ningún tipo de conflicto de interés asociado al desarrollo de la investigación y de la presentación de artículo. 


\section{Referencias}

Bédard, R. (2004). Los fundamentos del pensamiento administrativo y las prácticas administrativas - 2: la trilogía administrativa. AD-MINISTER, (4), 80-108 https://repository.eafit.edu.co/handle/10784/14100

Briggs T. (2018). Formal organizations, informal networks, and work flow: An Agent-Based Model. In Thomson R. et al. (Eds.) Social, Cultural, and Behavioral Modeling. SBP-BRiMS 2018. Lecture Notes in Computer Science, vol. 10899. Springer, Cham. https://doi.org/10.1007/978-3-319-93372-6_21

Capone, F., \& Lazzeretti, L. (2018). The different roles of proximity in multiple informal network relationships: Evidence from the cluster of high technology applied to cultural goods in Tuscany. Industry and Innovation, 25(9), 897-917. https://doi.org/10.1080/13662716.2018.1442713

Chambi, V. (2017). La interacción entre los trabajadores administrativos de la Universidad Nacional de Juliaca. Trabajo de grado para optar al título de licenciada en ciencias de la comunicación social, Universidad Nacional del Altiplano: Perú. http://repositorio.unap.edu.pe/handle/UNAP/10281

Chanlat, A. \& Bédard, R. (1990). La administración: una ética de la palabra. L'individu dans l'organisation, les dimensions oubliées. Québec: Université Lava.

Cross, O. (2018). Impact of informal groups on organisational performance. International Journal of Scientific Research and Management, 6(9), 686-694. https://doi.org/10.18535/ijsrm/v6i9.em04

De Faria, F. (2000). Desarrollo organizacional: enfoque integral. México, D.F.: Editorial Limusa, S.A. de C.V. Grupo Noriega Editores.

Gaete, F. \& Vásquez, J. (2008). Conocimiento y estructura en la investigación académica: una aproximación desde el análisis de redes sociales. REDES - Revista hispana para el análisis de redes sociales, 14(5), 1-33. https://www.r edalyc.org/articulo.oa?id=93101405

Granovetter, M. (1973). The strength of weak ties. American Journal of Sociology, 78, 1360-1380. https://doi.org/10 .1016/B978-0-12-442450-0.50025-0

Heller, R. \& Willower, D. (1968). Informal Structure in Elementary Schools. The Elementary School Journal, 69(2), 94-99. https://doi.org/10.1086/460484

Higuita, D. \& Leal, J. (2010). Microculturas y cultura organizacional: Construcción dialéctica en la organización. Revista de la Facultad de Ciencias Económicas, 18(1), 151-167. https://doi.org/10.18359/rfce.2286

Higuita, D. (2010). Discurso dominante de la dirección: refuerzo negativo a las relaciones humanas. Revista Innovar, 20(36). 67-78 https://www.redalyc.org/articulo.oa?id=81819028006

Higuita, D., Ballesteros, B., \& Pérez, P. (2010). Influencia de grupos informales en la gestión de Pymes industriales de Medellín. Lupa Empresarial, 12, 38-52. https://revistas.ceipa.edu.co/index.php/lupa/article/view/555

Johannessen, I., McArthur, P. \& Jonassen, J. (2015). Informal leadership redundancy: Balancing structure and flexibility in subsea operations. Scandinavian Journal of Management, 31(3), 409-423. https://doi.org/10.1016/j.scaman .2015 .01 .001

Johansen, Ó. (1992). Anatomía de la empresa. Una teoría general de las organizaciones sociales. México D.F.: Editorial Limusa y Grupo Noriega Editores.

Kegen, N. (2015). Cohesive subgroups in academic networks: Unveiling clique integration of top-level female and male researchers. Scientometrics, 103, 897-922. https://doi.org/10.1007/s11192-015-1572-z

Krackhardt, D. \& Hanson, J. (1993). Informal Network: The Company Behind The Chart. Harvard Business Review, 71(4), 104-111. https://hbr.org/1993/07/informal-networks-the-company-behind-the-chart

Krackhardt, D. \& Kilduff, M. (2002). Structure, culture and Simmelian ties in entrepreneurial firms. Social Networks, 24(3), 279-290 https://doi.org/10.1016/S0378-8733(02)00008-4

Krackhardt, D. \& Stern, R. (1988). Informal networks and organizational crises: An experimental simulation. Social Psychology Quarterly, 51(2), 123-140 https://doi.org/10.2307/2786835

Krackhardt, D. (1992). The Strength of Strong Ties: The importance of Philos in Organizations. In Nohria, N \& Eccles, R. Networks and Organizations. Structure, form and action. Boston: Harvard Bussiness School Press. 
Kuipers, K. (2009). Formal and Informal Network Coupling and Its Relationship to Workplace Attachment. Sociological Perspectives, 52(4), 455-479. https://doi.org/10.1525/sop.2009.52.4.455

Laubach, M. (2005). Consent, Informal Organization and Job Rewards: A Mixed Methods Analysis. Social Forces, 83(4), 1535-1565. https://doi.org/10.1353/sof.2005.0070

Lopes, B., Guimarães, R., Lima, M., Rocha, M., Heloisa, M., \& Pequeno, L. (2020). Analysis of informal social networks of friendship, trust and learning in a restaurant. Brazilian Journal of Management/Revista de Administração da UFSM, (13), 1255-1271. https://doi.org/10.5902/19834659 32397

Lozares, C. (1996). La teoría de redes sociales. Papers, (48), 103-126. https://doi.org/10.5565/rev/papers/v48n0.18 14

Luria, G. \& Berson, Y. (2013). How do leadership motives affect informal and formal leadership emergence? Journal of Organizational Behavior, 34(7), 995-1015. https://doi.org/10.1002/job.1836

Marino, J., Dabos, G., \& Gómez, M. (2020) Estructura social de la organización y toma de decisiones: efectos de los vínculos sociales en la implementación de iniciativas en una pyme del sector comercial 111. En Federico et al. (Eds.) 25 Reunión Anual Red Pymes Mercosur (pp. 670-681). http://redpymes.org.ar/wp-content/uploads/20 20/12/Libro-Resumen-25\%C2\%B0-Reuni\%C3\%B3n-Anual-Red-Pymes-Mercosur.pdf\#page=670

Marqués, P., Farrerons, L., Arias, N., \& Quiroga, E. (2012). Las relaciones informales: un valor añadido en la gestión de personas. Enfermeria Global, 11 (26), 310-323. https://doi.org/10.4321/S1695-61412012000200020

Martínez, R. (2018). Formulación, modelamiento y simulación de un modelo generativo de la cultura organizacional en la Universidad del Valle basado en sistemas multiagente. Tesis Doctoral. Cali: Universidad del Valle.

Mena, A. \& Viloria, J. (2015). Aproximación teórica a los grupos informales desde la Administración y la Psicología: una mirada hacia sus dinámicas e influencia en las organizaciones. Proyecto de pregrado. Santa Marta: Universidad del Magdalena.

Miner, R. (2013). Informal Leaders. Journal of Leadership, Accountability and Ethics, 10(4), 57-61.

Muñoz-Rojas, D., Vargas-Ortiz, L., \& Latta-Arias, C. (2018). Estructura del clima organizacional en algunas universidades públicas del norte de Colombia. IPSA Scientia, Revista Cientifica Multidisciplinaria, 3(1), 17-22. https://latinjournal.org/index.php/ipsa/article/view/921/699

Poveda, G. I. (2020). Influencia de las redes sociales informales en los proyectos empresariales de la Unidad de Emprendimiento e Innovación (UEI) de la Universidad Nacional de Colombia, sede Bogotá. Trabajo de grado. Bogotá: Universidad Nacional de Colombia.

Sangrà, A. \& Wheeler, S. (2013). Nuevas formas de aprendizaje informales: ¿O estamos formalizando lo informal? Revista de Universidady Sociedad del Conocimiento, 10(1), 107-115. http://dx.doi.org/10.7238/rusc.v10i1.1689

Santos, R. F. (1989). El concepto de Red Social. Reis: Revista Española de Investigaciones Sociológicas, (48), 137-152. https://doi.org/10.2307/40183465

Venkataramani, V., Zhou, L., Wang, M., Liao, H., \& Shi, J. (2016). Social networks and employee voice: The influence of team members' and team leaders' social network positions on employee voice. Organizational Behavior and Human Decision Processes, 132, 37-38. https://doi.org/10.1016/j.obhdp.2015.12.001

Wang, Y., Andrew, G. \& Pavel, A. (2016). Do manufacturing firms need informality in ERP post-implementation? A study of Chinese manufacturing sites. Journal of Manufacturing Technology Management, 27(1), 100-123. http s://doi.org/10.1108/JMTM-09-2015-0077

White, L., Currie, G. \& Lockett, A. (2016). Pluralized leadership in complex organizations: Exploring the cross network effects between leadership influence and informal network relations. The Leadership Quarterly, 27(2), 280-297 https://doi.org/10.1016/j.leaqua.2016.01.004

Williams, A., Verwoord, R., Beery, T., Dalton, H., McKinnon, J., et al. (2013). The power of social networks: A model for weaving the scholarship of teaching and learning into institutional culture. Teaching \& Learning Inquiry: The ISSOTL Journal, 1(2), 49-62. https://doi.org/10.2979/teachlearninqu.1.2.49

Winston, S. (2005). The role of informal networks in higher education: The tipping point for a line officer's success or failure. Doctoral Thesis. https://search.proquest.com/docview/305388394?accountid=4151 
Zapata, Á. (2008). Análisis y diseño organizacional. De la estructura funcional a la organización vacía. Cali: Universidad del Valle.

Zehua, T. (2018). Informal networks and knowledge sharing in organizations: Case study of GR Group. Thesis doctoral. http://hdl.handle.net/10071/17099

\section{Apéndice}

\section{Anexos}

TABLA A1

Caracterización miembros de la organización informal, dependencia A

\begin{tabular}{|c|c|c|}
\hline Miembro & Tipo & Caracterizaciones \\
\hline $\begin{array}{l}\text { Sujeto } \mathrm{S} 10 \\
\text { (Director } \\
\text { dependencia) }\end{array}$ & Enlace $^{1}$ & $\begin{array}{l}\text { Este sujeto recibió cinco referenciaciones que lo caracterizan así: } \\
\text { S3: "atento, buena persona, alegría, confiable, respetuoso". } \\
\text { S25: "Inteligente, pragmático". } \\
\text { S19: "Respetuoso, amigable, líder, sociable, responsable". } \\
\text { S17: "Confiable, profesional, buen amigo, capaz, integro, buen humor". } \\
\text { S11: "Responsable y justo". }\end{array}$ \\
\hline $\begin{array}{l}\text { Sujeto S2 } \\
\text { (Coordinador) }\end{array}$ & Enlace & $\begin{array}{l}\text { Este sujeto recibió seis referenciaciones que lo caracterizan así: } \\
\text { S21: "amabilidad, responsabilidad, respeto, compromiso, madurez". } \\
\text { S17: "profesional, inteligente, educada, incondicional, buena amiga, sincera, } \\
\text { correcta". } \\
\text { S9: "Compañera de maestría, rango de edad similar". } \\
\text { S6: "Es una persona llena de virtudes, excelente amiga y compañera". } \\
\text { S10: "Inteligente, segura, amable, cordial". } \\
\text { S3: "Amigable, honesta, prudente, sincera". }\end{array}$ \\
\hline $\begin{array}{l}\text { Sujeto S24 } \\
\text { (Coordinador) }\end{array}$ & Integral $^{2}$ & $\begin{array}{l}\text { Este sujeto recibió tres referenciaciones, de las cuales dos son de tipo } \\
\text { bidireccional. } \\
\text { S7: "Prudente". } \\
\text { S16: "Conocimiento de los procesos administrativos y académicos". } \\
\text { S25: "Buen trabajador". }\end{array}$ \\
\hline $\begin{array}{l}\text { Sujeto S1 } \\
\text { (Coordinador) }\end{array}$ & Integral & $\begin{array}{l}\text { Este sujeto recibió tres referenciaciones, una de las cuales proviene del } \\
\text { miembro enlace S2. } \\
\text { S6: "buena compañera y amiga". } \\
\text { S2: "es una amiga, neutral y una madre, siempre pendiente de su familia". } \\
\text { S25: "apasionada". }\end{array}$ \\
\hline $\begin{array}{l}\text { Sujeto S8 } \\
\text { (Docente) }\end{array}$ & Integral & $\begin{array}{l}\text { Este miembro presenta tres referenciaciones, una de las cuales es de otro } \\
\text { miembro integral, los sujetos que lo referencian lo caracterizan como: } \\
\text { S16: "conocimiento, prudencia y sabiduría". } \\
\text { S25: "metódico". } \\
\text { S24: (no hizo descripción). }\end{array}$ \\
\hline $\begin{array}{l}\text { Sujeto S4 } \\
\text { (Docente) }\end{array}$ & Integral & $\begin{array}{l}\text { Este sujeto presenta tres referenciaciones, una es del miembro enlace S10. } \\
\text { S10: "divertido, alegre, confiado, servicial". } \\
\text { S16: "conocimiento de los procesos académico". } \\
\text { S21: "responsabilidad, honestidad, compromiso, profesionalismo, respeto". }\end{array}$ \\
\hline $\begin{array}{l}\text { Sujeto S14 } \\
\text { (Docente) }\end{array}$ & Integral & $\begin{array}{l}\text { Este sujeto recibió tres referenciaciones y una de ellas proviene de otro } \\
\text { miembro integral S4 y un miembro enlace S2. } \\
\text { S4: "sinceridad, honesta, seria". } \\
\text { S6: "buena compañera y amiga". } \\
\text { S2: "es una gran amiga, siempre dispuesta a escucharnos". }\end{array}$ \\
\hline $\begin{array}{l}\text { Sujeto S12 } \\
\text { (Docente) }\end{array}$ & Integral & $\begin{array}{l}\text { Este sujeto lo referencian tres individuos que lo caracterizan como: } \\
\text { S9: "compartimos gustos y preferencias, compañero de la maestría". } \\
\text { S19: "amigable, respetuoso, sociable, amable, responsable". } \\
\text { S13: "sincera, respetuosa, agradable, tranquilo y positivo". }\end{array}$ \\
\hline
\end{tabular}

Fuente: elaboración propia. 
TABLA A2

Caracterización miembros de la organización informal, dependencia $B$

\begin{tabular}{|c|c|c|}
\hline Miembro & Tipo & Caracterizaciones \\
\hline $\begin{array}{l}\text { Sujeto S42 } \\
\text { (Docente tipo 2) }\end{array}$ & Integral & $\begin{array}{l}\text { Este sujeto recibió cinco referenciaciones, que lo caracterizan como: } \\
\text { S40: "Amable, respetuosa y sincera". } \\
\text { S26: "Inteligente, creativa y buen sentido del humor". } \\
\text { S34: "Seriedad, ética y amable". } \\
\text { S45: "Carisma, trayectoria y don de gente". } \\
\text { S29: "Responsable y capaz". }\end{array}$ \\
\hline $\begin{array}{l}\text { Sujeto } \mathrm{S} 29 \\
\text { (Director } \\
\text { Dependencia) }\end{array}$ & Integral & $\begin{array}{l}\text { Este miembro muestra cuatros referencias que lo caracterizan así: } \\
\text { S40: "Honesta, sincera y responsable". } \\
\text { S26: "Trabajadora, inteligente y sencilla". } \\
\text { S42: "Proactivo, seguro de sí mismo, honesto y servicial". } \\
\text { S45: "Dinamismo, capacidades de gestión, y don de gente". }\end{array}$ \\
\hline $\begin{array}{l}\text { Sujeto S45 } \\
\text { (Docente tipo 2) }\end{array}$ & Integral & $\begin{array}{l}\text { Este individuo recibió tres referenciaciones que lo caracterizan de } \\
\text { forma breve, de la siguiente manera: } \\
\text { S26: "Sinceridad, inteligente". } \\
\text { S34: "Disciplina". } \\
\text { S28: "Cariño y respeto". }\end{array}$ \\
\hline $\begin{array}{l}\text { Sujeto S38 } \\
\text { (Asistente } \\
\text { dependencia B) }\end{array}$ & Integral & $\begin{array}{l}\text { Este sujeto presenta cinco referenciaciones que la caracterizan así: } \\
\text { S46: "es amigable, cariñosa y respetuosa". } \\
\text { S27: "sincera, amable y trabajadora". } \\
\text { S31: "me anima a confiar en ella su carácter, su forma de hablar y } \\
\text { tratar a los demás, y sobre todo su forma única de ser". } \\
\text { S33: "carisma". } \\
\text { S29: "activa, productiva". }\end{array}$ \\
\hline $\begin{array}{l}\text { Sujeto } \mathrm{S} 27 \\
\text { (Asistente otra } \\
\text { dependencia) }\end{array}$ & Integral & $\begin{array}{l}\text { Este sujeto recibió tres referenciaciones que la definen así: } \\
\text { S31: "amorosa, sensible, humilde y sencillez". } \\
\text { S38: "sincera, amable, respetuosa, cariñosa y sensible". } \\
\text { S46: "social, gentil y sobre todo humilde". }\end{array}$ \\
\hline $\begin{array}{l}\text { Sujeto S31 } \\
\text { (Asistente otra } \\
\text { dependencia) }\end{array}$ & Integral & $\begin{array}{l}\text { Este miembro presenta tres referencias que lo caracterizan así: } \\
\text { S46: "responsable y porque su persona da para confiar en ella". } \\
\text { S27: "Carácter fuerte, trabajadora, amable, colaboradora". } \\
\text { S38: "espontanea, sincera, fuerte". }\end{array}$ \\
\hline $\begin{array}{l}\text { Sujeto } \mathrm{S} 46 \\
\text { (Asistente otra } \\
\text { dependencia) }\end{array}$ & Integral & $\begin{array}{l}\text { Este sujeto recibió el mínimo de tres referenciaciones que la definen } \\
\text { de la siguiente manera: } \\
\text { S31: "valentía, humor, carácter y humildad". } \\
\text { S38: "sincera, espontanea, carismática y segura". } \\
\text { S27: "amable, colaboradora, trabajadora". }\end{array}$ \\
\hline
\end{tabular}

Fuente: elaboración propia.

\section{Notas}

* Artículo de investigación.

\section{Licencia Creative Commons CC BY 4.0}

Para citar este artículo: Mena, A., García, O, \& Zapata, A. (2021). Caracterización de los vínculos en la organización informal de una Institución de Educación Superior. Cuadernos de Administración, 34. https:/ /doi.org/10.11144/Javeriana.cao34.cvoiies 